Espacio y Desarrollo No 33, 2019, pp. 67-89 (ISSN 1016-9148)

https://doi.org/10.18800/espacioydesarrollo.201901.004

\title{
EL TRABAJO FEMENINO INDÍGENA EN LA ECONOMÍA AGRÍCOLA FAMILIAR EN LA AMAZONÍA SUR DEL ECUAdOR
}

\author{
Viviana Buitrón Cañadas \\ Asociación Geográfica del Ecuador \\ viviana.buitronc@gmail.com \\ Maria Fernanda López Sandoval \\ FLACSO Ecuador \\ maflopez@flacso.edu.ec
}

Fecha de recepción: 2 de mayo de 2019

Fecha de aceptación: 12 de junio de 2019

\section{Resumen}

El presente artículo analiza la situación del trabajo femenino en los hogares de Miazi y Shaime, dos comunidades shuar en la Amazonía sur ecuatoriana, en un contexto de colonización y de reciente apertura a mercados locales. Se observan además las implicaciones del trabajo de las mujeres en la agro-biodiversidad y en la alimentación de sus familias, así como la valoración subjetiva sobre su participación. Los resultados muestran principalmente dos espacios de producción agrícola en las fincas familiares de los entrevistados: el aja o huerta para autoconsumo y los cultivos de comercialización. Esta caracterización se hace en función del rol que las mujeres cumplen en cada espacio y de la carga de trabajo que tienen en el proceso productivo. Además, se presentan algunos extractos de entrevistas de varios actores locales y comunitarios que contextualizan las condiciones desiguales y de sobre-explotación sobre las cuales se desarrolla el trabajo femenino. Este análisis revela que, aunque invisible, el trabajo de las mujeres es central en una economía agrícola de mercado en desarrollo en estos ecosistemas ecológicamente sensibles. Palabras clave: trabajo femenino, agricultura familiar, cultivos de mercado, Amazonía

\section{Indigenous female work in the household-farm economy in the Southern Ecua- dorian Amazon}

\section{Abstract}

This article analyzes the situation of female work among the households in Miazi and Shaime, two Shuar communities in the Southern Ecuadorian Amazon, in a context of colonization and 
recent local market opening. The implication of women's work in terms of agro-biodiversity and their family diet are also observed, as well as the subjective assessment of their participation. Findings depict mainly two spaces of agricultural production in the interviewees' household farms: the aja or garden for self-consumption and the market crops. This characterization is based on the role that women fulfill in each space and the workload they have in the production process. In addition, some excerpts from interviews to various local and community actors are presented; they contextualize the uneven conditions and over-exploitation on which women's work develops. This analysis reveals that, although invisible, women's work is central to a developing agricultural market economy in these ecologically sensitive ecosystems.

Keywords: Female work, family farming, market crops, Amazon

\section{INTRODUCCIÓN}

En América Latina, la biorregión de la Amazonía es una de las más importantes a nivel ecológico regional y global (de Jong, Borner, Pacheco,Pokorny, Sabogal, Benneker, 2011). Asimismo, esta área constituye una de las últimas fronteras de expansión del modelo capitalista a través de la extracción de recursos naturales, la implantación de relaciones mercantiles y, todavía, la colonización agraria (Coy, Ruiz Peyré y Obermayr, 2017; López, 2000; Rivero y Cooney, 2010; Tsing 2005, p. 28). Estos procesos están perturbando los ciclos naturales, modificando los contextos productivos y culturales de poblaciones indígenas (Lalander y Kröger, 2016) y han generado deterioro ambiental a diferentes escalas, como la deforestación, considerada uno de los problemas más graves de la región (Martino, 2007; Shukla, Nobre y Sellers, 1990). Los impactos de estos cambios han recibido incluso menos atención en cuanto a la formas de organización del trabajo rural amazónico, dentro del proceso de expansión capitalista y globalización (Arango, 2004, p. 3). Si bien existen algunos estudios sobre la proletarización de la población amazónica en el marco de la extracción minera y petrolera (cf. Wasserstrom y Bustamante, 2015), las relaciones de trabajo agrícola que surgen cuando la economía de mercado se ancla en estas fronteras colonas han sido poco estudiadas. Este escenario podría entenderse bajo la aproximación del debate agrario (López y Carrión, 2018; Martínez y North, 2007) en contextos de nueva ruralidad (Kay, 2009). Este enfoque, para Latinoamérica, debate acerca de los modos de producción y los escenarios de dominación que contextualizan la situación de la población rural en el actual período de globalización neoliberal desde perspectivas étnicas y de género, y donde la cuestión ambiental también es importante. Dentro de estas discusiones, la reestructuración del trabajo de la población local, sobre todo el de las mujeres indígenas, trae consigo un impacto no solo en el acrecentamiento de procesos de degradación ecológica sino también en el propio sostenimiento de sus hogares. 
En Ecuador, la reestructuración del trabajo indígena ha sido discutida especialmente en el contexto andino (cf. Barsky, 1984; Korovkin, 2002). Sobre el trabajo de las mujeres rurales se conoce que su inserción en las actividades productivas es heterogénea (León y Deere, 1997). Sin embargo, la naturalización de su rol reproductivo ha opacado el aporte productivo que realizan y que se ha traducido en una mayor carga global de trabajo en relación a la de los hombres rurales (Vásconez, 2012, p. 83). Esta situación implica para las mujeres tener menos tiempo para sí mismas, para su educación y otras actividades de tipo social (Radcliff, 2014, p. 22). Estudios más recientes señalan la importancia del trabajo indígena femenino en el agro-negocio del brócoli (Yumbla, 2014) y en la producción florícola del Ecuador desde la década de 1980 (Coffey, 2016; Vásconez, 2012). No obstante, existe poco análisis sobre las relaciones de género en los varios aspectos del mundo rural (Kay, 2007, p. 44), con una evidente desatención al trabajo rural femenino indígena, tanto en la intervención de la política pública como en la investigación académica.

En este contexto, la situación actual del trabajo agrícola en la Amazonía del Ecuador y, en particular, del trabajo agrícola femenino ha sido aún menos revisada. El acceso a mercados y el uso de mano de obra para la agricultura comercial dentro del ciclo de vida de los hogares han sido abordados en estudios sobre manejo de recursos, deforestación y cambios en el uso del suelo por el avance de la colonización (e.g. Barbieri, Bilsborrow y Pan, 2005; Bilsborrow, Barbieri y Pan, 2004; Hutchison, 2016). Sin embargo, la dinámica de inserción de la población indígena amazónica en mercados agrícolas no ha producido en general investigaciones significativas, a pesar de que, como lo indica López y Sierra (2011), la intensificación de la agricultura en territorios amazónicos indígenas es similar a la de los colonos en el marco de la producción orientada al mercado. En el caso de las mujeres indígenas de esta región, su posición en los mercados de producción y trabajo ha presentado desventajas notorias en relación a los hombres indígenas o las mujeres no-indígenas (Radcliff, 2014).

Para el caso de la Amazonía sur del Ecuador, las transformaciones espaciales y en la estructura de trabajo están relacionadas a la colonización desde la década de 1970 (cf. Mera y Montańo, 1984; Rudel y Horowitz, 1996) que han convertido el bosque en espacios agrícolas o ganaderos de pequeños productores vinculados a mercados locales (e.g. Gerique, 2011; Rudel, Bates y Machinguiashi, 2002). Este proceso en el Alto Nangaritza es reciente y ocurre en el marco de una tardía inserción en la economía nacional (Buitrón, 2017) y del rápido desarrollo de infraestructura vial desde el año 2000 (Gerique, López y Pohle, 2017), lo que ha repercutido notablemente en el uso de suelo y la cobertura vegetal. Así, la cobertura boscosa en la zona ha disminuido del $94 \%$ en 1978 al 71\% en el año 2013 (Buitrón, 2018). Al igual que en otros sitios de la Amazonía (cf. Barbieri et al., 2005), patrones de usos del suelo con requerimientos más intensivos de mano de obra reemplazan la cobertura boscosa. En esta zona es notable 
la presencia, como cultivo orientado al mercado, de la especie Solanum quitoensis, localmente conocida como naranjilla. En estas áreas, así como en los espacios de agricultura de subsistencia, el trabajo femenino resulta fundamental (Radcliff, 2014, p. 16).

En este contexto, este artículo tiene como objetivos 1) caracterizar el trabajo de las mujeres indígenas amazónicas del Alto Nangaritza en dos espacios de producción agrícola: en la huerta (aja, en idioma shuar) y en los cultivos de comercialización, y 2) analizar las implicaciones de la distribución del trabajo femenino en la economía familiar y en la seguridad alimentaria de sus comunidades. El análisis se enfoca en la economía agrícola familiar y en la división sexual del trabajo, para centrar la reflexión sobre el rol de la fuerza de trabajo femenina indígena en un marco de colonización y apertura a mercados agrícolas.

La agricultura familiar ocupa un espacio importante en el análisis de lo rural en América Latina por ser la base del trabajo agrícola y de la soberanía y la seguridad alimentaria, y por contribuir en el mantenimiento ecológico y cultural (Van der Ploeg, 2015). Si bien el modelo teórico de la agricultura familiar se caracteriza por la producción agrícola de subsistencia para cubrir las necesidades internas de alimentación del hogar, incorpora también, en diferente medida, la producción para el mercado (cf. Jiménez, 2007; Walker, Perz, Caldas y Silva, 2002). Sin mantener una lógica enteramente capitalista, la agricultura familiar combina entonces estrategias domésticas de producción, tanto mercantiles y no mercantiles (Martínez, 2014).

Por otro lado, la división sexual del trabajo es variable, dependiendo de regiones o actividades, o ambigua y flexible en contextos de desarrollo del sistema capitalista (Deere y León, 1982). Sin embargo, esta división es observable a través de las prácticas agrícolas que permiten identificar concretamente las relaciones sociales entre los sexos (Garcés, 2017, p. 15). De manera general, las mujeres se concentran, sobre todo, en el trabajo reproductivo, pero también realizan actividades productivas; ambas, como trabajo no remunerado (Benería, 2006, p. 11). La atribución de tareas se relaciona con modos específicos de producción y edictos culturales específicos (Arizpe, 1986, p. 59). Estas actividades, productivas o reproductivas, son un proceso continuo que reemplaza y sostiene la fuerza de trabajo y el tejido social que, dentro del sistema capitalista, son indispensables para la reproducción del capital (cf. Gammage y Orozco, 2008).

\section{CASO DE ESTUdio Y MÉTODOS}

\section{Área de estudio: El Alto Nangaritza}

El área conocida localmente como el Alto Nangaritza está localizada al sureste de la provincia amazónica de Zamora Chinchipe (Figura 1). Es parte de la depresión de Amotape-Huancabamba (Beck y Richter, 2008), la cual funciona geomorfológicamente 
como un puente para la migración de la biodiversidad con un importante componente de endemismo (Freile, Moscoso y Félix, 2010). Por estas condiciones ecológicas excepcionales, el Alto Nangaritza concentra varias áreas de conservación, como el Bosque Protector Alto Nangaritza (2002), la Reserva Natural Maycu (2013), la Reserva Comuntaria Los Tepuyes (2014) y la Reserva Ecológica Cerro Plateado (2010). Esta última, junto con los Parques Nacionales Podocarpus y Yacuri, forman parte de las zonas núcleo de la Reserva de Biósfera Podocarpus-El Cóndor (2007). El área de estudio justamente es parte de la zona de transición de esta reserva.

\section{Figura 1. El Alto Nangaritza}

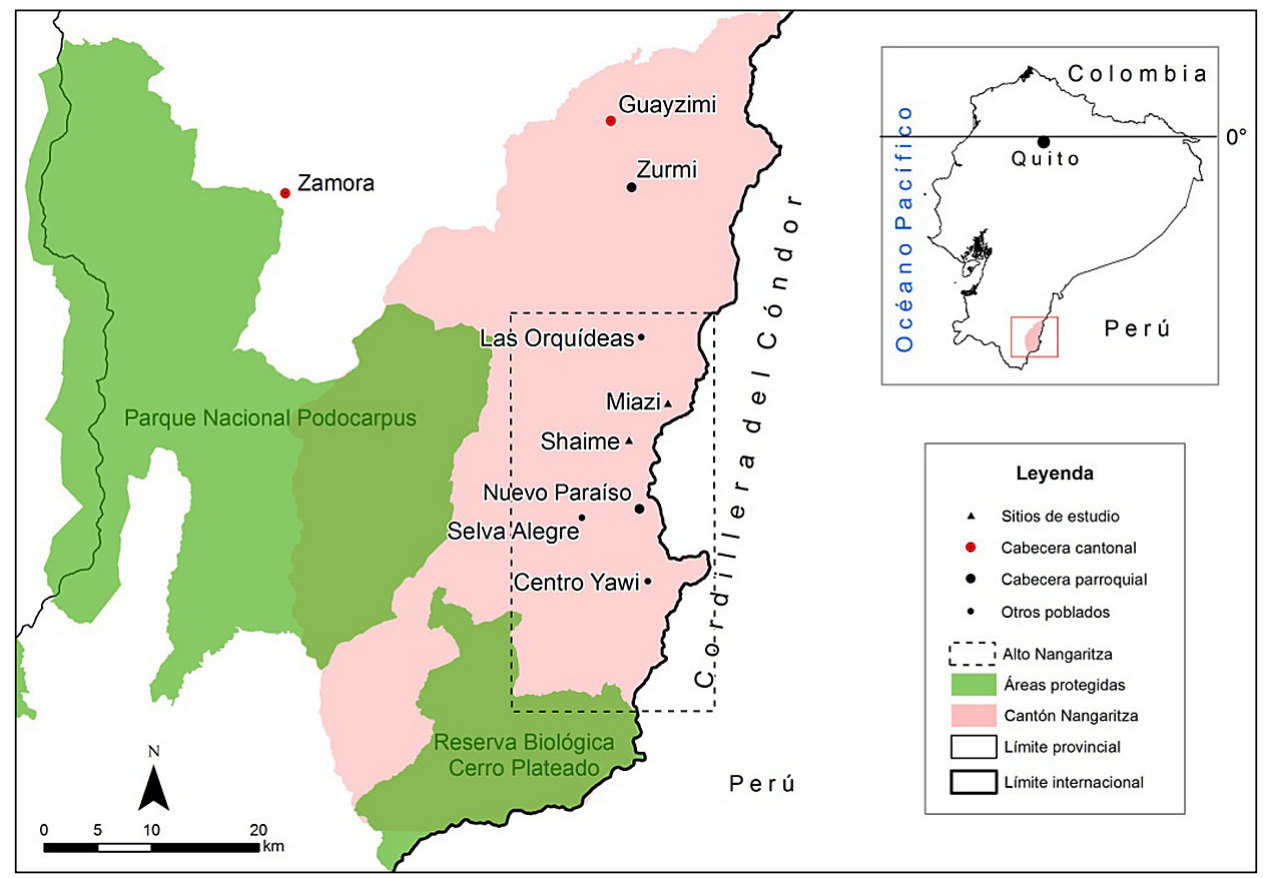

Fuente: IGM, 2010; INEC, 2010; MAE, 2015, datos de descarga libre. Elaboración propia.

El censo del año 2010 señala que la zona tenía 1578 habitantes (Instituto Nacional de Estadísticas y Censos - INEC, 2010), de los cuales, 814 eran hombres $(51,6 \%)$ y 764 mujeres $(48,4 \%)$, con tasas de crecimiento poblacional intercensales desde la década de 1990 más altas que las calculadas a nivel nacional. Por ejemplo, para el período 2001-2010, la tasa de crecimiento total en la zona fue de 3,8\% en el área a diferencia de $1,95 \%$ a nivel nacional (Villacís y Carrillo, 2012, p. 11).

Los procesos de migración y colonización a esta área han definido su diversidad étnico-cultural: shuar y colonos (saraguros y mestizos que migraron desde los Andes). 
La mayoría, 899 individuos (57\%), son de la etnia shuar, habitantes históricos amazónicos. El resto de la población se autoidentifica como mestiza (30\%), saraguro (4\%) y otras etnias (aproximadamente 9\%). Generalmente, los shuar viven en comunidades, conocidas también como centros, y mantienen títulos comunitarios de su tierra. La Asocición Tayunts de segundo grado, según la estructura política indígena ecuatoriana, agrupa a las comunidades shuar del Alto Nangaritza (Shaime, Miazi, Wampiashuk, Yawi, Yayu, SaarEntza, Chumpias, Nayump, Shakay y Napints). Por otro lado, colonos mestizos y saraguros llegaron a la zona como beneficiarios de programas de colonización promovida por el Estado y formalizados a través de títulos de propiedad individual otorgados por diversas agencias estatales desde la década de 1970 (Ministerio de Agricultura y Ganadería - MAG, 1977). Los colonos mantienen la tenencia individual de sus terrenos y se agrupan en asociaciones o cooperativas de tipo productivo y comercial, formas organizativas que, hasta inicio de la década de 1990, fueron requisitos para solicitar tierra al Estado en el marco de la colonización.

\section{Métodos}

El análisis presentado en este artículo es parte de un proyecto de investigación más amplio sobre las relaciones existentes entre cambios de uso de suelo desde perspectivas interétnicas y multiescalares (cf. Buitrón, 2018). Para el análisis aquí presentado, se trabajó con la información cualitativa, recogida en varias visitas al campo entre 2014 y 2016 en los centros Miazi (148: 77 mujeres y 71 hombres) y Shaime (126: 69 mujeres y 57 hombres) (Buitrón, 2018). Ambos son reconocidos formalmente como comunidades shuar, aunque en Miazi también hay casos de matrimonios interétnicos.

Los participantes fueron seleccionados siguiendo criterios del muestreo de bola de nieve o método de referencia en cadena, útil para identificar posibles voluntarios en poblaciones de difícil acceso. Este método consiste en preguntar entre los mismos individuos que participan o por otros posibles voluntarios con características importantes para la investigación (Jensen y Shumway, 2010). La información levantada provino de entrevistas semiestructuradas, técnica que se basa en una guía de preguntas (Bernard, 2006), y que, en este caso, se enfocó en el contexto local del área, la historia de colonización, los cambios en el uso de recursos y las prácticas agrícolas y las interrelaciones sociales.

Los informantes especializados ${ }^{1}(\mathrm{~N}=8)$ fueron siete hombres y una mujer entre autoridades locales, expertos técnicos y adultos mayores. También se realizaron cuestionarios multiobjetivo que son herramientas que establecen una lista de preguntas como

\footnotetext{
Los nombres de las/los informantes especializadas/os fueron anonimizados y codificados en secuencia desde SI-01 hasta SI-08. Si algún extracto de las entrevistas se cita en el texto, este será especificado con el código correspondiente.
} 
categorías analíticas para facilitar una posterior codificación de las respuestas, y cuyo fin es profundizar en temáticas específicas (Briones, 2002). En este caso, se puso especial énfasis en la organización interna de los hogares con respecto de los usos de suelo en sus fincas, las prácticas agrícolas y la distribución del trabajo, tanto en el espacio del aja como en los cultivos de comercialización. Esta herramienta se aplicó a informantes clave/cabezas de hogar o a sus parejas $(\mathrm{N}=13)$, contando con ocho hombres y cinco mujeres ${ }^{2}$. A nivel metodológico, tener el punto de vista de los hombres sobre el rol de las mujeres enfatiza la visibilidad - o no- del trabajo femenino en la economía familiar y las implicaciones subjetivas de su participación. Además, cabe recalcar que para las respuestas que concernían al aja, los hombres entrevistados solicitaron, en la mayoría de los casos, la asistencia de su esposa.

En cuanto al análisis, las grabaciones de las entrevistas semiestructuradas fueron transcritas y sometidas a un análisis de contenido, a partir de una codificación deductiva. El esquema de esta codificación se establece previamente con base en temas emergentes desde, por ejemplo, la revisión bibliográfica o reportes previos (Bernard, 2006). La información de los cuestionarios fue tabulada y analizada según el uso de suelo reportado por los hogares. Con esto, se definieron las características de esos espacios de producción, con la participación femenina como enfoque. Dadas las características de la muestra y las necesidades del análisis, las interpretaciones de los resultados se apoyaron también en la revisión bibliográfica y la documentación oficial disponible de los centros shuar, y se pusieron en relación a la información de la población colona para enmarcar las respuestas en contextos de colonización, como es este caso.

\section{Resultados y discusión}

El aja en la economía y cultura indigena shuar a través del trabajo femenino

El aja (Figura 2) ha sido históricamente el espacio central en la estructuración de la vida shuar (Carrillo, 2012; Montero y Crespo, 1989). En conjunto con el bosque y la vida humana, el aja comprendía una integralidad, y su disposición espacial era alrededor de la casa, según lo reportado (005Sh). En este sentido, los límites de uso de los recursos estaban - y aún están en gran medida- determinados por la capacidad propia de la naturaleza para absorber los impactos de sus intervenciones y regenerarse.

\footnotetext{
2 Los códigos de los 18 hogares participantes se especifican como «0XXSh» si fue una entrevista conducida en Shaime, o como «0XXMz» si tuvo lugar en Miazi. Si algún extracto de las entrevistas se cita en el texto, este será especificado con el código correspondiente.
} 
Figura 2. Aja shuar en Shaime. Al frente: plantas de yuca (Manihot esculenta)

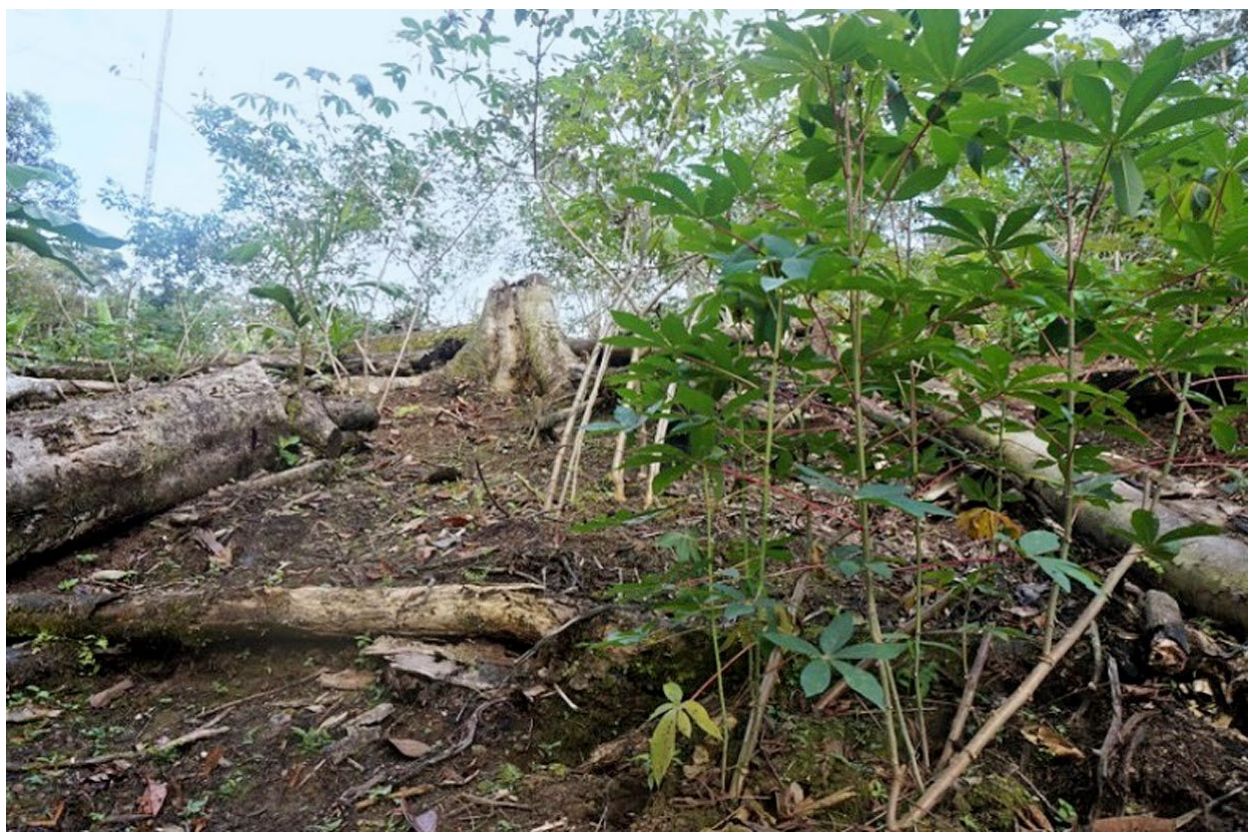

Fotografía: Viviana Buitrón, 2014.

Actualmente, la organización del aja, aunque ha sufrido varios cambios, depende aún de los ciclos de cultivo itinerante (shifting cultivation) (Houghton, Lefkowitz y Skole, 1991, p. 180). De acuerdo a la información levantada, el indicador del buen rendimiento del aja es el cultivo de yuca (Manihot esculenta) (024Sh). La superficie dedicada a este espacio depende de las necesidades de los miembros del hogar. De las encuestas realizadas se estima un tamaño promedio de aja de 0,6 ha, que representa una pequeña proporción del tamaño promedio reportado de las fincas shuar en territorio comunal (alrededor de 40,5 ha). A pesar de su reducida extensión, todos los 13 hogares shuar en Miazi y Shaime tienen al menos un espacio en las fincas familiares destinado a este uso (ver Tabla 1). El aja es el espacio de trabajo femenino para la producción de alimentos de autoconsumo, principalmente. Como lo manifestó un informante en Shaime (SI-05), el aja es considerada como su propio "mercado», manteniéndose de esta forma como el espacio que garantiza la seguridad y soberanía alimentaria de los hogares indígenas del área de estudio.

La información empírica del estudio, indica que, al igual que en las chacras de las comunidades andinas (Deere y León, 1982), las actividades en el aja tienen una clara distribución sexual que es incluso imitada por los hogares colonos. Los datos de las encuestas indican que hay un consenso entre la pareja sobre las condiciones óptimas 
del terreno donde se vaya a levantar una nueva aja; el hombre hace el desbroce inicial del bosque para ello y la mujer tiene el rol central de mantener el aja a largo plazo y de realizar todas las actividades agrícolas de cuidado, como limpieza, cosecha o selección de semillas. Esto concuerda con las observaciones de Pohle (2008) en cuanto a la transmisión de conocimientos sobre la ecología de siembra y cosecha en este espacio desde la línea materna. Además, se indicó que los cánticos rituales a Nunkui (el espíritu femenino en el aja), que aún se practican, se enseñan solamente a las hijas mujeres (SI-05).

Tabla 1. Caracterización de las ajas shuar reportadas en Miazi y Shaime

\begin{tabular}{ll}
\hline Número de hogares & $\begin{array}{l}\text { Todos los 13 hogares shuar tienen al menos un aja. } \\
\text { Cuatro de los 13 hogares shuar tienen dos ajas. } \\
\text { Uno de los } 13 \text { hogares shuar tiene tres ajas. }\end{array}$ \\
\hline Tamańo total & 7,8 ha en las fincas de los 13 hogares shuar \\
\hline Período de uso & Máximo ocho años, aunque depende del cuidado de la mujer \\
\hline Distancia en tiempo & Indeterminado: desde cinco minutos hasta dos horas a pie \\
\hline Persona encargada del cuidado & Esposa \\
\hline Razones de localización & $\begin{array}{l}\text { Elección del cabeza de hogar, usualmente sobre lusaras antiguas } \\
\text { (tierras en barbecho) }\end{array}$ \\
& $\begin{array}{l}\text { Lo más cerca del lugar de vivienda, pero lejos de los niños } \\
\text { Buenas condiciones de suelo, especialmente para la yuca }\end{array}$ \\
\hline
\end{tabular}

Frecuencia de las visitas/cuidado Diario: cuatro hogares

Tres veces por semana: cuatro hogares

Dos veces por semana: dos hogares

Una vez por semana: dos hogares

Origen de la técnica empleada $\quad$ Principalmente es conocimiento aprendido del lado materno.

Productos mencionados yuca/mama (Manihot esculenta), piña (Ananas comosus), camote/inchi (Ipomoea batatas), plátano/champiar (Musa sp.), kenkelpapatuyo (Dioscorea trifida), kumpia (Renealmia alpinia), maní (Arachis hypogaea), papaya (Carica papaya), papa china (Colocasia esculenta), sangolpelma (Xanthosoma sp.), naranjilla silvestre (Solanum quitoense), chiki (Maranta ruiziana), zapallo (Cucurbita ficifolia), namuka (Sicana odorifera), barbasco (Lonchocarpus nicou), palma de chonta (Bactris gasipaes), piripiri (Cyperus spp.), kanz'e (Aerva sanguinolenta), ushu (Caladium bicolor), granadilla dulce (Passiflora ligularis), badea (Passiflora quadrangularis), maíz (Zea mays), fréjol (Phaseolus cf. vulgaris), caña de azúcar (Saccharum officinarum), frutales varios

Consumo Exclusivo para el hogar: 10 hogares Más del $70 \%$ para el hogar: tres hogares

Razones de abandono del aja Capacidad de trabajo y mantenimiento

* En Perú, estas áreas se denominan purmas

Fuente: Trabajo de campo en 2015 y 2016; información adaptada de Buitrón (2018). 
El trabajo de la mujer en este espacio para la conservación de las semillas ha permitido que se haya desarrollado por generaciones una amplia agrobiodiversidad en estas condiciones amazónicas. Las más de 24 especies nombradas están acordes a inventarios (etno) botánicos llevados a cabo anteriormente entre la población shuar del sur del Ecuador, las cuales superan a las reportadas en las huertas de los colonos (cf. Federación Interprovincial de Centros Shuar - FICSH, 1976, p. 66; Gerique, 2011; Pohle y Gerique, 2008).

Por otro lado y a pesar de que actualmente las ajas se ubican a distancias variables de los poblados, la mayoría de las mujeres informó que sí va más frecuentemente a cuidar el aja si el tiempo de recorrido a pie desde sus casas es corto (hasta 20 minutos). Sin embargo, a la exclusividad del cuidado de este espacio como causa para los desplazamientos diarios de las mujeres se le han sumado las actividades relacionadas con el mercado que requieren también del trabajo femenino para su realización. En este sentido, se reportó que en las familias que poseían ganado con requerimientos de atención diaria y donde la mujer se vincula de alguna forma, ella sí que va al aja todos los días, pero porque "queda de camino» (008Mz) al lugar donde están los pastos.

Además de alimentar al hogar a través del trabajo femenino, el aja, el cultivo de la yuca y la elaboración de la chicha ${ }^{3}$, en conjunto con la caza y la pesca, son parte importante de la cultura shuar (SI-02). Estas son actividades que se comparten al interior del hogar y en la vida social y comunitaria. Debido a estas características, el aja, como espacio del trabajo femenino, se constituye en un elemento material central para la soberanía alimentaria y para la producción y reproducción cultural shuar.

\section{Los cultivos de comercialización y la participación femenina}

Aunque la apertura de pastos y la crianza de ganado son más antiguas y más extensas en superficie $e^{4}$, los cultivos de comercialización constituyen la actividad económica más ampliamente aceptada entre la población indígena shuar de esta área (SI-02). Esta actividad les genera ingresos más rápidos y regulares, de los que ha dependido su economía sobre todo desde los primeros años de los 2000 cuando el cultivo de naranjilla (Solanum quitoense) (Figura 3) se popularizó (SI-04). Sobre esto se reportó que:

"[Se eligió la naranjilla] [p]orque tiene un mercado amplio y es apetecida por los comerciantes informales o intermediarios. Aunque se tale mucho el bosque pero es la actividad que trae mejores recursos.» (SI-08)

\footnotetext{
3 La chicha es una bebida fermentada tradicional hecha a base de yuca (Manihot esculenta) para las festividades, mingas o consumo diario. Conectada a su importancia cultural, a nivel alimentario, la chicha puede reemplazar una comida completa o ser un líquido refrescante e hidratante para los días calurosos y de trabajo. 4 Según las entrevistas, de los 13 hogares shuar entrevistados de Shaime y Miazi, solo siete hogares dijeron tener pastos en sus fincas. De ellos, solo cuatro tienen ganado. Todo esto a pesar de que cuentan con las superficies más grandes de terreno para los patrones de uso extensivos de los pastos a comparación de los otros grupos étnicos.
} 
Se pueden incluso identificar razones culturales detrás de la preferencia de esta actividad agrícola por sobre la ganadera, según las entrevistas:

"La mujer no puede ver ganado en el shuar (...) le tiene miedo. Y el shuar casi no consume ganado, hay un porcentaje que no toma la leche, no come la carne, solo es para vender.» (SI-07)

La Tabla 2 muestra las principales características de las áreas dentro de las fincas shuar destinadas a cultivos de comercialización, según la información levantada. De los trece hogares entrevistados, diez tienen por lo menos un área en su finca destinada a los cultivos de mercado. De estos, cinco tienen dos superficies para estos fines. La superficie promedio de esta área por finca de los diez hogares que afirmaron tener este uso de suelo fue de alrededor de 1,4 ha.

Tabla 2. Caracterización de los cultivos de comercialización en las fincas shuar reportadas de Miazi y Shaime

\begin{tabular}{ll}
\hline Número de hogares & $\begin{array}{l}\text { 10 de los 13 hogares shuar tienen al menos un área destinada a cultivos } \\
\text { de comercialización. } \\
\text { Cinco de los 13 hogares shuar tienen dos áreas destinadas a cultivos de } \\
\text { comercialización. }\end{array}$ \\
\hline Tamaño total & $\begin{array}{l}\text { 14 ha aproximadamente en las fincas de los 10 hogares shuar que tienen } \\
\text { cultivos de comercialización }\end{array}$ \\
\hline Período de uso & Si la parcela es de naranjilla, el máximo es de tres cosechas (una por año). \\
\hline Distancia en tiempo & Indeterminado: usualmente menos de 30 minutos a pie \\
\hline $\begin{array}{l}\text { Persona encargada del } \\
\text { cuidado }\end{array}$ & $\begin{array}{l}\text { Solo esposo: cinco hogares } \\
\text { Principalmente esposo: dos hogares } \\
\text { Principalmente esposa: dos hogares }\end{array}$ \\
\hline Solo esposa: un hogar \\
\hline $\begin{array}{l}\text { Razones de localización } \\
\text { Frecuencia de las visitas/ }\end{array}$ & $\begin{array}{l}\text { Preferiblemente en suelo virgen si es para la naranjilla } \\
\text { Cercano al sitio de venta o a la carretera }\end{array}$ \\
\hline Origen de la técnica & $\begin{array}{l}\text { Depende de la fase de crecimiento de los cultivos: desde una vez al mes } \\
\text { hasta todos los días }\end{array}$ \\
\hline empleada & $\begin{array}{l}\text { De otros shuar con experiencia anterior en estos cultivos } \\
\text { De los colonos o de talleres técnicos }\end{array}$ \\
\hline Productos mencionados & $\begin{array}{l}\text { Solo naranjilla: nueve hogares } \\
\text { Plátano: 1 hogar }\end{array}$ \\
\hline Consumo & Exclusivamente para el mercado \\
\hline Razones de abandono de los \\
cultivos de comercialización
\end{tabular} \begin{tabular}{l} 
Por desgaste del suelo y disminución de las cosechas \\
\hline
\end{tabular}

Fuente: Trabajo de campo en 2015 y 2016; información adaptada de Buitrón (2018).

Así como la naranjilla se ha constituido en una parte importante del ingreso financiero de la población local, también ha generado un alto impacto tanto ambiental como 
socioeconómico. Este cultivo es exigente en cuanto a sus requerimientos ecológicos para su producción y por ciertas condiciones económicas que el hogar debe tener para mantener este tipo de cultivos. Un buen rendimiento de naranjilla necesita preferiblemente suelo recién desmontado o suelo recuperado después de largos períodos de lusara. Considerando estas condiciones de cultivo, este uso de suelo se convertiría en el principal driver de deforestación local (Buitrón, 2018). Por otro lado, la siembra de naranjilla requiere de una alta inversión financiera para costear la producción como monocultivo: semillas, plaguicidas y otros insumos químicos para el control de malezas. De hecho, todos los diez hogares que practican estas actividades han utilizado fertilizantes químicos alguna vez, unos incluso desde la primera siembra a inicios de los 2000. Para esto, seis hogares reportaron haber recurrido a préstamos a instituciones financieras (cooperativas) para cubrir esos gastos.

Acerca de la técnica utilizada, el modo de cultivo se desarrolló a partir de la experiencia traída por otros shuar desde aguas abajo del río Nangaritza donde ya se había iniciado con este cultivo en los años 70 (SI-04) y de las escasas capacitaciones técnicas — sin seguimiento a largo plazo — dadas por instituciones gubernamentales relacionadas con el tema agroproductivo (SI-05). Las actividades agrícolas que se llevan a cabo en este espacio están estandarizadas y son comunes entre nativos y colonos para lograr rendimientos que respondan a las exigencias del mercado.

Figura 3. Clasificación de la naranjilla para la venta en Miazi

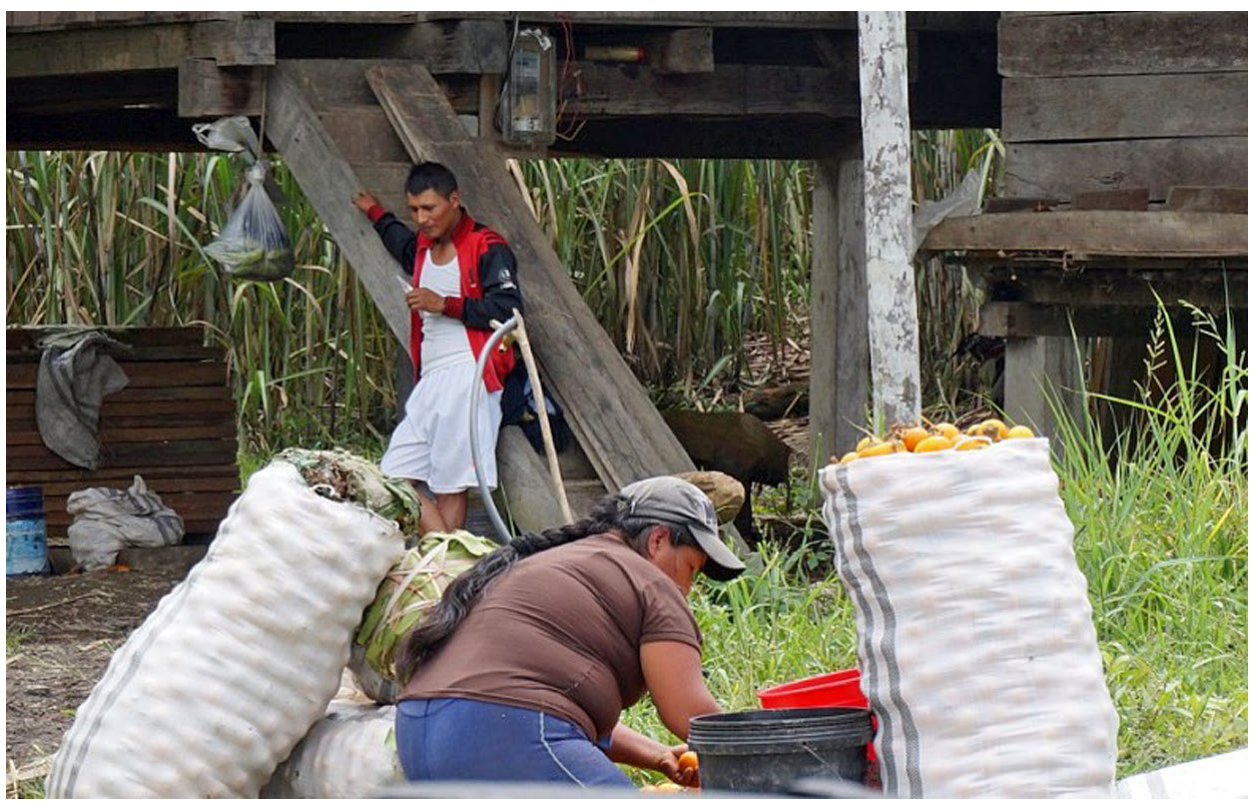

Fotografía: Viviana Buitrón, 2015. 
A diferencia del aja, en los cultivos de comercialización la división sexual del trabajo es flexible, pues depende de algunos condicionantes socioeconómicos como la disponibilidad de contratar mano de obra (peones), o la precaución de que las mujeres en gestación no trabajen en los cultivos durante los períodos de fumigación. De cualquier manera, la mitad de hogares participantes en este análisis indicaron que en este cultivo hay, en diferente grado, participación femenina. Su intervención productiva es más intensa a mayor vinculación del esposo con otras actividades (e.g. extracción de madera, servicio público) o ante la ausencia de este, situación que fue observada también por Hutchison (2016) en comunidades de la Amazonía norte ecuatoriana. Este trabajo agrícola productivo se adiciona entonces a las tareas de las mujeres en el aja y en el hogar.

Acerca del consumo de la producción de este cultivo al interior del hogar, los shuar entrevistados, a diferencia de los colonos, indicaron no consumir naranjilla, debido a la cantidad de agrotóxicos usados en la fumigación. En este sentido, toda la fuerza de trabajo empleada en estos cultivos, incluyendo el trabajo femenino, es capitalizada por el mercado. Si bien los ingresos permiten cubrir créditos bancarios, comprar insumos o cubrir gastos de educación de los/las hijos/as, esta producción no contribuye a la seguridad alimentaria del hogar.

En la comercialización, el trabajo indígena comúnmente no es reconocido, y el trabajo femenino es totalmente invisibilizado. Los shuar entrevistados en general admiten tener dificultades al negociar pagos justos por su cosecha con los intermediarios (SI-05, SI-08), de quienes depende la variación semanal de los precios. Los informantes shuar indican que estas dificultades se deben a la poca experiencia tanto con el uso del dinero como medio de transacción como con la valorización del trabajo agrícola remunerado (SI-07). Por otro lado, al no existir una asociación, la comercialización vuelve a los productores financieramente vulnerables para exigir precios justos. Las ganancias, aunque sean marginales, se quedan íntegramente en los hombres, sin el reconocimiento del aporte femenino, a pesar de que la mujer se integra de manera activa a la fase de producción. Sobre esto se dijo que:

"Al momento que ingresó el recurso [financiero] y se empieza a administrar el recurso, antes solo el hombre administraba la plata, la mujer poco administraba.» (SI-07)

De acuerdo a las explicaciones de Vizcarra-Bordi, Lutz y Ramírez-Hernández (2013, p. 212), en el caso de comunidades indígenas mexicanas, esta intensificación de las tareas en los hombres es de carácter productivo, por lo tanto debe ser reconocida financieramente. En cambio, cuando estas actividades migran hacia las mujeres lo hacen en realidad como actividades domésticas no remuneradas. Entonces, la producción hacia el mercado, como lo argumenta Campillo (2000), estaría asentando sus bases en la sobre-explotación del trabajo familiar en la misma unidad de producción 
agrícola, beneficiándose de ese trabajo no remunerado de las mujeres en condiciones desiguales entre los géneros. Esta desigualdad en la distribución de tareas y beneficios pone en cuestionamiento las ideas de complementariedad y relaciones recíprocas entre sociedades rurales en formación a partir de la colonización, como lo observó también Garcés (2017) en el caso rural colombiano.

\section{Las implicaciones del trabajo femenino en la economia familiar y la seguridad alimentaria}

Con el análisis de la información empírica de campo, se identificaron dos grandes impactos a nivel material y simbólico del trabajo femenino dentro de la economía familiar y la seguridad alimentaria de los hogares: a) cambios en la agrobiodiversidad del aja y en la alimentación del hogar, y b) cambios en la valoración simbólica del trabajo de la mujer. La cuestión del trabajo femenino y la invisibilización del mismo y sus implicaciones se relacionan con, entre otros factores, la restricción del derecho de las mujeres a la tierra y el tutelaje masculino aún persistente, como se discute más adelante.

Tal como se reportó en la sección anterior, la mujer se ha integrado a las actividades productivas de mercado, aunque al interior del hogar la distribución de tareas no ha cambiado y las responsabilidades de cuidado y producción del aja siguen siendo atribuidas a las mujeres. En este marco, el mantenimiento del aja para autoconsumo se ha complicado porque parte del tiempo del trabajo femenino es usado ahora en los cultivos del comercialización. La carga de trabajo adicional, comparada con la de sus parejas, resulta difícil de sobrellevar al mismo tiempo y en distintos espacios de producción, impactando en la diversidad agrícola de sus ajas. Especies cultivadas, como el kenke (Dioscorea trifida) o la namuka (Sicana odorifera), típicamente usadas en la cocina shuar, se encuentran con menos frecuencia (SI-08), y el conocimiento etnobotánico local asociado a esas plantas se ha perdido, como lo señaló también Gerique (2011). Estas observaciones concuerdan, además, con las estimaciones de disminución de biodiversidad en la provincia de Zamora Chinchipe, donde se encuentra el Alto Nangaritza. Según un reporte de la Federación Provincial de la Nacionalidad Shuar de Zamora Chinchipe - FEPNASH-ZCH (2008, p. 34), alrededor de cuarenta especies de plantas manejadas por los shuar han desaparecido o están siendo cultivadas con menos regularidad que antes.

Otro impacto en la agrobiodiversidad está relacionado con las nuevas necesidades financieras del hogar que demandan que algunos productos del aja deban comercializarse también, aunque en menor medida que la naranjilla. La yuca (Manihot esculenta) y el plátano (Musa sp.), por su alta aceptación en el mercado, empiezan a dominar espacialmente la parcela en detrimento del espacio para otros cultivos y del uso del tiempo de trabajo de las mujeres.

Además de los efectos de la reestructuración del tiempo de trabajo femenino en el sistema de la finca shuar en la agrobiodiversidad y en la alimentación en términos 
materiales, hay también un impacto simbólico acerca de su trabajo alrededor de la alimentación. Durante las entrevistas, la pérdida de cultivos tradicionales fue una queja permanente entre los hombres shuar de mayor edad, quienes culpaban a las mujeres y a su "falta de trabajo» por las transformaciones en su alimentación y, por ende, por la desaparición de su cultura y territorialidad concentradas en la práctica del aja (cf. Buitrón, 2018).

"En este caso la mujer shuar ya no prepara [el kengu']. Antes la mujer shuar cogía un kengu', lo asaba y le brindaba al esposo y era una fruta apetitosa. Ahora ya no lo preparan. Si se trae no lo usan y hasta se pudre. Entonces ya no se consume. Ahora si no hay, papa, arroz, fideos, cebolla ya no quieren cocinar, todo va cambiando.» (SI-08)

Este tipo de enunciados de los hombres contradicen la perspectiva de las mujeres, para quienes los cambios alimenticios son a causa de la preferencia de los hombres ante la opción de productos procesados, empacados y altamente calóricos (e.g. arroz, atún enlatado, fideos). En un contexto de colonización, este tipo de alimentos se vuelven económicamente asequibles para las familias que ahora ya cuentan con un ingreso financiero relacionado con el mercado agrícola o con el trabajo fuera de finca. Debido a que las actividades de las mujeres no dejan de ser de carácter reproductivo, cuando se les culpa por los cambios en la alimentación, se les reclama precisamente el incumplimiento de su responsabilidad de cuidadora del hogar y del aja como su «espacio sagrado", desestimando la sobrecarga de tareas a cumplir diariamente.

Esta carga, además de ser invisible en el contexto familiar, también lo es entre la comunidad y para el Estado que tampoco reconocen las características del trabajo femenino. Por ejemplo, el desaparecido Consejo de Desarrollo de las Nacionalidades y Pueblos del Ecuador - Codenpe aprobó los estatutos de creación de Shaime (Centro Shuar Shaim, 2008), en el cual constaban los oficios de los/las 59 fundadores de este lugar. En la nómina, los registros de los hombres aparecen actividades como «agricultor», «auxiliar» o «profesor». Sin embargo, la categoría de «quehaceres domésticos» (QQDD) es general y exclusiva entre las mujeres. El ser agricultor constituye, incluso para este organismo estatal, un oficio que se identifica en los hombres, mientras que las actividades agrícolas intensivas que también realizan las mujeres son naturalizadas como parte de sus obligaciones dentro del hogar. Según las observaciones de Baquerizo (2014) en la costa ecuatoriana, esta naturalización del trabajo femenino significa que se subordina al de los hombres adquiriendo una posición secundaria con respecto a este.

Conectado con el no-reconocimiento de las implicaciones del trabajo femenino en la economía familiar y la seguridad alimentaria a nivel material y simbólico, el ejercicio de los derechos de las mujeres sigue siendo restringido. Esto ahonda más el estado invisible del trabajo femenino indígena. El acceso a la tierra es limitado en general para las mujeres, situación que tiene raíces histórico-estructurales. Según Radcliff (2014, p. 17), esto sucede desde el mismo momento de la abolición del sistema de hacienda, 
cuando a las mujeres no se les reconoció ningún derecho para la redistribución de tierras, sino al hombre como cabeza del hogar de producción agrícola. Esta situación se ha venido replicando y es observable a nivel comunitario en el área de estudio. Si bien Shaime y Miazi son territorios comunales e indivisibles en su constitución, el acceso a la tierra está asentado sobre una base étnica y sigue siendo transmisible en derecho de uso principalmente para los hombres, incluso si estos se casan con una mujer de fuera de la comunidad (SI-01). A la mujer, en cambio, este derecho le es negado directamente, mucho más si se une con alguien de otro grupo étnico. Sobre esto, se dijo en dos entrevistas que:

"Hay mestizaje. Si, existe. Hay parejas que se casan con hispanos [colonos] (...). Si es mujer tiene que irse con el marido y entonces no tienen ningún derecho. Si [el marido] quiere ser socio tiene que ser comprobado que les gusta vivir en la organización bajo juramento. Eso es caso especial.»(SI-O1)

Además:

«(...) pero si [las mujeres] se casan con alguien de otra nacionalidad, pueden volver, pero ya no tienen derechos. Pueden trabajar y cumplir con todo igual que los socios, pero el marido ya no tiene derechos en la comunidad.» (SI-08)

No obstante, si un colono se casa con una mujer de la comunidad podría convertirse en un miembro de la comunidad shuar si la asamblea así lo decidiese. De esta manera, los colonos, para quienes el acceso a la tierra es fundamental en términos de herencia y producción, pueden lograr una convivencia relativamente estable con los shuar y minimizar las tensiones por temas de propiedad. Esto se observó particularmente en Miazi, donde hay matrimonios interétnicos entre saraguros o mestizos con mujeres shuar, lo cual ha coadyuvado a la aceptación de la presencia colona. Por otro lado, este tipo de acuerdos locales generalmente entre hombres, miembros de hecho y de derecho de las comunidades, ha permitido el desarrollo del sistema campesino ganadero de los saraguros en la Amazonía (cf. Buitrón, 2017).

Así, a varios niveles, las mujeres han sido sistemáticamente despojadas del derecho al acceso a la tierra como medio de producción, sino es a través de los hombres. En este sentido, la cuestión de la tierra, como lo destacó también Garcés (2017), toma entonces una posición central sobre la condición de las mujeres.

Aparte de la restricción del disfrute de ciertos derechos, la invisibilidad del trabajo femenino se relacionaría con el hecho de que las mujeres están consideradas aún bajo el tutelaje de los hombres: primero de su padre y luego de su esposo. Si bien las uniones sentimentales y las alianzas matrimoniales han ido transformándose en su conformación, el papel de subordinación de la mujer ha permanecido intacto.

"Cuando hija de un tío. Digamos tú tienes un hermano. Tu hija, el hijo de tu hermano (...) Entonces ese hijo es el dueño de esa chica (...) Cuando nace mujercita ya queda dueño 
(...) Entonces qué pasaba? Desde ese tiempo ya comenzaba a mantener este joven, mantener al suegro, a la suegra (...) con pescado, carne, lo que sea. Entonces le daba a la suegra para que dé de comer. Y asi era, caminaba 5 años, 6 años y asi ellas (...) sentian como su padre [al futuro esposo].» (SI-06)

Estructuras patriarcales persisten aún entre la población local. En el imaginario colectivo, sobre todo en contextos indígenas y rurales, el rol de las mujeres no solo que pertenece al ámbito doméstico, sino que es sobre todo objeto de control masculino (Sandner Le Gall, 2013, p. 307). Morrison (2013, p. 371), haciendo referencia a la teoría de dominación legitimada de Weber, argumenta que esa autoridad patriarcal está basada en derechos naturalizados para los hombres de lo que siempre ha sido hecho y reconocido a través de edictos debido a reglas de larga data.

En este marco de reestructuración del trabajo de las mujeres en función de las nuevas condiciones del mercado, los tiempos a dedicar en cada actividad impactan en el sostenimiento la alimentación de sus familias y en la valoración subjetiva hacia el trabajo femenino en las comunidades. Actividades agrícolas más intensivas y una consecuente deforestación generan además otras maneras de relacionarse con la naturaleza. Esto forma parte, además, de un proceso de desposesión cultural en favor de sistemas económica y culturalmente «más exitosos» (Montero y Crespo, 1989) que, despojándole de su autonomía, (re)organizan los espacios y el trabajo al interior de los hogares. Esta situación pone en conflicto las prácticas agrícolas desarrolladas por las mujeres a lo largo del tiempo dentro de la agricultura de subsistencia con las que les exige la agricultura moderna (Radcliff, 2014, p. 14). No obstante, sea como productivo o reproductivo dentro del hogar y la unidad de producción, la importancia del trabajo femenino ha permanecido invisible por cuestiones estructurales, de dominación y subordinación, a pesar de que su contribución al mantenimiento de la fuerza de trabajo y a la reproducción social es central (Benería, 2006, p. 10; Deere y León, 1982).

\section{ReFLeXiones Finales}

En los hogares de las comunidades shuar del Alto Nangaritza, se identificó que el papel de las mujeres no está limitado solamente a la esfera doméstica. En ausencia parcial o total de trabajo de los hombres y en una economía de mercado, las mujeres shuar están asumiendo roles económico-productivos, los cuales están en complementación y a la vez en conflicto con sus actividades reproductivas y de cuidado. Esta participación del trabajo femenino, aunque invisibilizada, está ciertamente cambiando la distribución de la fuerza de trabajo en el sistema agrícola familiar y contribuye al aceleramiento de la producción agrícola para el mercado, ya que existe una migración del tiempo de trabajo en el aja hacia actividades de mercado. 
Si bien las actividades agrícolas se adjudican comúnmente a los hombres con una consecuente extensificación e intensificación de uso, sobre todo en dirección a pastos y cultivos de comercialización (cf. Barbieri y Carr, 2005), una consideración del género en estos procesos (Geist, McConnell, Lambin, Moran, Alves y Rudel, 2010), dentro de un marco de demanda de mano de obra para el mercado, pone en cuestión el rol del trabajo femenino como factor en la deforestación no solo a nivel ecológico. La atención en el género es central desde un punto de vista productivo y económico, y también desde una perspectiva económico-cultural y de valorización simbólica del trabajo femenino, ya que la dualidad bosque-aja es la base material de los medios de vida shuar para su seguridad alimentaria y de la transmisión intergeneracional del conocimiento etnobotánico.

Este análisis del trabajo femenino entre los shuar muestra la necesidad de abordajes más amplios sobre la economía agrícola familiar dentro del debate agrario actual. Estas discusiones deben ampliarse además hacia otras áreas rurales, como las amazónicas, donde reestructuraciones en el modo de producción agrícola están sucediendo a partir de procesos relativamente recientes de colonización y de avance del mercado. Con el reconocimiento de las condiciones desiguales en las que se da el trabajo femenino, se debe pensar luego, como lo plantea León $(2009$, p. 6), en otra manera de organizar la producción y la reproducción con condiciones justas y coherentes con los límites de la naturaleza y de la fuerza de trabajo de las mujeres que garantice a la vez los medios de vida de sus familias y comunidades.

\section{REFERENCIAS}

Arango, L. (2004). Mujeres, trabajo y tecnologías en tiempos globalizados. Cuaderno $\mathrm{N}^{\circ} 5$. Bogotá: Centro de Estudios Sociales, Universidad Nacional de Colombia. Recuperado de http://bdigital.unal.edu.co/39970/1/Mujerestrabajoytecnologia.pdf

Arizpe, L. (1986). Las mujeres campesinas y la crisis agraria en América Latina. Nueva Antropología, 8(30), 57-65. Recuperado de http://www.redalyc.org/articulo. oa?id $=15903004$

Baquerizo, E. (2014). «La mujer invisible»: Producción y reproducción en el agro de la costa interior ecuatoriana (Tesis de maestría). Departamento de Sociología y Estudios de Género, FLACSO. Quito. Recuperado de https://repositorio.flacsoandes.edu.ec/ bitstream/10469/6484/2/TFLACSO-2014EBC.pdf

Barbieri, A., Bilsborrow, R. y Pan, W. (2005). Farm Household Lifecycles and Land Use in the Ecuadorian Amazon. Population and Environment, 27(1), 1-27. https://doi. org/10.1007/s11111-005-0013-y

Barbieri, A. y Carr, D. (2005). Gender-specific out-migration, deforestation and urbanization in the Ecuadorian Amazon. Global and Planetary Change, 47(2), 99-110. https:// doi.org/10.1016/j.gloplacha.2004.10.005 
Barsky, O. (1984). Acumulación campesina en el Ecuador. Los productores de papa del Carchi. Quito: Flacso. Recuperado de https://biblio.flacsoandes.edu.ec/libros/digital/56750.pdf Beck, E. y Richter, M. (2008). Ecological aspects of a biodiversity hotspot in the Andes of southern Ecuador. En R. Gradstein et al. (eds.), The tropical mountain forest. Patterns and processes in a biodiversity hotspot (pp. 195-217). Gotinga, Alemania: Universitätsverlag Göttingen (Biodiversity and ecology series, v. 2).

Benería, L. (2006). Trabajo productivo/reproductivo, pobreza y políticas de conciliación. Género y políticas públicas desafíos de la equidad. Nómadas, 24, 8-21. Recuperado de http://nomadas.ucentral.edu.co/nomadas/pdf/nomadas_24/24_1B_Trabajoproductivo.pdf

Bernard, H. (2006). Research methods in anthropology. Qualitative and quantitative approaches. 4th ed. Lanham, MD: AltaMira Press. Recuperado de http://www. cycledoctoralfactec.com/uploads/7/9/0/7/7907144/\%5Bh._russell_bernard\%5D_ research_methods_in_anthropol_bokos-z1_1_.pdf

Bilsborrow, R., Barbieri, A. y Pan, W. (2004). Changes in Population and Land Use over Time in the Ecuadorian Amazon. Acta Amazonica, 34(4), 635-647. https://doi. org/10.1590/S0044-59672004000400015

Briones, G. (2002). Metodología de la investigación cuantitativa en las ciencias sociales. Santafé de Bogotá: Icfes (Programa de especialización en teoría, métodos y técnicas de investigación social, 3). Recuperado de https://metodoinvestigacion.files.wordpress. com/2008/02/metodologia-de-la-investigacion-guillermo-briones.pdf

Buitrón, V. (2017). Colonización y acuerdos locales en la consolidación del sistema campesinoganadero saraguro en la Amazonía sur del Ecuador. Eutopia Revista de Desarrollo Económico Territorial, 12, 103-119. https://doi.org/10.17141/eutopia.12.2017.2911

Buitrón, V. (2018). Land-uselland-cover change (LUCC) in the context of an agricultural frontier in the southern Ecuadorian Amazon: A multiscale and interethnic perspective (Tesis doctoral, no publicada). Instituto de Geografía, Friedrich-Alexander Universität Erlangen-Nürnberg.

Campillo, F. (2000). El trabajo doméstico no remunerado en la economía. Nómadas, 12, 98-115. Recuperado de http://www.redalyc.org/pdf/1051/105115263011.pdf

Carrillo, W. (2012). Construcción identitaria shuar a la luz de los procesos de modernización - Estudio de caso - Transformaciones del núcleo familiar a través de la educación popular (Tesis de maestría). Antropología, Flacso. Quito. Recuperado de https://repositorio. flacsoandes.edu.ec/bitstream/10469/5988/2/TFLACSO-2012WCD.pdf

Centro Shuar Shaim (2008). Nómica de socios fundadores de Centro shuar Shaim. Shaim: Autor.

Coffey, G. (2016, enero 26). En el nombre de la rosa: La industria florícola y sus espinas. En La línea de fuego. Recuperado de https://lalineadefuego.info/2016/01/26/en-elnombre-de-la-rosa-la-industria-floricola-y-sus-espinas-por-por-gerard-coffey/ 
Coy, M., Ruiz Peyré, F. y Obermayr, C. (2017). South American resourcescapes: geographical perspectives and conceptual challenges. Die Erde, 148(2-3), 93-110. https://doi. org/10.12854/erde-148-41

de Jong, W., Borner, J., Pacheco, P., Pokorny, B. y Sabogal, C. (2011). Los bosques amazónicos en la encrucijada: presiones, respuestas y desafíos. En W. de Jong y G. Mery (eds.), Desafíos de los bosques amazónicos y oportunidades para el manejo forestal comunitario, CIAS Discussion Paper 20, IUFRO Occasional Paper 25 (pp. 2-30). Vantaa: Finnish Forest Research Institute. Recuperado de https://www.researchgate.net/publication/256980120_Los_ bosques_amazonicos_en_la_encrucijada_Presiones_respuestas_y_desafios

Deere, C. y León, M. (1982). Producción campesina, proletarización y la división sexual del trabajo en la zona andina. En M. León (ed.), Debate sobre la mujer en América Latina y el Caribe: discusión acerca de la unidad producción-reproducción (pp. 115-132). Bogotá: ACEP.

Federación Interprovincial de Centros Shuar - FICSH (1976). Solución original al problema actual. Sucúa: Autor.

Federación Provincial de la Nacionalidad Shuar de Zamora Chinchipe - FEPNASH-ZCH. (2008). Plan de Gobierno 2008-2013. Zamora: Autor.

Freile, J., Moscoso, P. y Félix, C. (eds.) (2010). La magia de Los Tepuyes del Nangaritza: una guí para conocer a sus habitantes. Quito: Conservación Internacional Ecuador.

Gammage, S. y Orozco, M. (2008). El trabajo productivo no remunerado dentro del hogar: Guatemala y México. Serie Estudios y perspectivas 103. México D.F.: CEPAL. Recuperado de https://repositorio.cepal.org/bitstream/handle/11362/4882/S0800810_ es.pdf? sequence $=1$ \&isAllowed $=y$

Garcés, D. (2017). Colonización campesina, división sexual del trabajo y acceso de las mujeres a la tierra: Aproximaciones al caso de las mujeres rurales de Tillavá. Mediaciones, 19, 10-31. https://doi.org/10.26620/uniminuto.mediaciones.13.19.2017.10-31

Geist, H., Mcconnell, W., Lambin, E., Moran, E., Alves, D. y Rudel, T. (2010). Causes and Trajectories of Land Use/Cover Change. En E. Lambin y H. Geist (eds.), Landuse and land-cover change. Local processes and global impacts (pp. 41-70). Berlin: Springer (The IGBP Series). https://doi.org/10.1007/3-540-32202-7_3

Gerique, A. (2011). Biodiversity as a resource: Plant use and land use among the Shuar, Saraguros, and Mestizos in tropical rainforest areas of southern Ecuador (Tesis doctoral). Instituto de Geografía, Friedrich-Alexander Universität Erlangen-Nürnberg. Recuperado de http://www.opus.ub.uni-erlangen.de/opus/volltexte/2011/2752/

Gerique, A., López, M. F. y Pohle, P. (2017). Sitting on a ticking bomb? A political ecological analysis of conservation conflicts in the Alto Nangaritza Valley, Ecuador. Die Erde, 148(2-3), 134-149. https://doi.org/10.12854/erde-148-44

Houghton, R., Lefkowitz, D.S. y Skole, D. (1991). Changes in the landscape of Latin America between 1850 and 1985. I. Progressive loss of forests. Forest Ecology and Management, 38(3-4), 143-172. https://doi.org/10.1016/0378-1127(91)90140-Q 
Hutchison, H. (2016). Deforestation and Female Participation in Natural Resource Management: Comparing Case Studies in Indigenous and Colonist Communities in the Province of Napo, Ecuador (Tesis de maestría). Departamento de Desarrollo, Ambiente y Territorio, FLACSO. Quito. Recuperado de https://repositorio.flacsoandes.edu.ec/ bitstream/10469/9798/2/TFLACSO-2016HCH.pdf

Instituto Nacional de Estadísticas y Censos - INEC (2010). Censo nacional de población y vivienda 2010 [datos censales y GIS database]. REDATAM Process. Quito: Autor.

Instituto Geográfico Militar - IGM (2010). Shapefiles de información básica y temática [GIS database]. Quito. Recuperado de http://www.geoportaligm.gob.ec/portal/index.php/ descargas/cartografia-de-libre-acceso/

Jensen, R. y Shumway, J. (2010). Sampling Our World. En B. Gomez y J. P. Jones III (eds.), Research Methods in Geography (pp. 77-90). Malden, Oxford, West Sussex: Wiley-Blackwell.

Jiménez, E. (2007). La diversificación de los ingresos rurales en Bolivia. Iconos. Revista de Ciencias Sociales, 29, 63-76. https://doi.org/10.17141/iconos.29.2007.236

Kay, C. (2007). Algunas reflexiones sobre los estudios rurales en América Latina. Iconos. Revista de Ciencias Sociales, 29, 31-50. https://doi.org/10.17141/iconos.29.2007.230

Kay, C. (2009). Estudios rurales en América Latina en el período de globalización neoliberal: ¿una nueva ruralidad? Revista Mexicana de Sociología, 71(4), 607-645. Recuperado de http://www.scielo.org.mx/pdf/rms/v71n4/v71n4a1.pdf

Korovkin, T. (2002). Comunidades indígenas, economía del mercado y democracia en los Andes ecuatorianos. Quito: CEDIME, IFEA, Ediciones Abya Yala. Recuperado de https:// digitalrepository.unm.edu/cgi/viewcontent.cgi? referer=https://www.google.com/\&h ttpsredir $=1 \&$ article $=1036 \&$ context=abya_yala

Lalander, R. y Kröger, A. M. (2016). Extractivismo y derechos étnico-territoriales de jure y de facto en latinoamérica. Observatorio del Desarrollo, 23, 1-22. Recuperado de https://helda.helsinki.fi//bitstream/handle/10138/231710/LalanderKrogerExtractivismosDerechosEtnicosTerritoriales16.pdf?sequence=1

León, M. (2009). Cambiar la economía para cambiar la vida desafíos de una economía para la vida. En A. Acosta y E. Martínez (comps.), El buen vivir (pp. 63-74). Quito: Ed. Abya-Yala. Recuperado de http://base.socioeco.org/docs/acosta-martinezel_buen_vivir.pdf

León, M. y Deere, C. (1997). La mujer rural y la reforma agraria en Colombia. Cuademos de Desarrollo Rural, 38-39, 7-23. Recuperado de https://revistas.javeriana.edu.co/ index.php/desarrolloRural/issue/view/198

López, A. (2000). Environmental Change, Social Conflicts and Security in the Brazilian Amazon: Exploring the Links (Tesis doctoral). Departamento de Ciencias Políticas, Universidad de Oslo. Recuperado de https://www.duo.uio.no/bitstream/ handle/10852/14230/4355.pdf?sequence $=1$ 
López, S. y Sierra, R. (2011). A resource demand model of indigenous production: The Jivaroan cultivation systems of Western Amazonia. Agricultural Systems, 104, 246-457. https://doi.org/10.1016/j.agsy.2010.10.003

López, M. F. y Carrión, A. (2018). Geografía, economía y territorios rurales en América Latina: presentación del dossier. Eutopía, 14, 7-22. https://doi.org/10.17141/eutopia.14.2018.3771

Martínez, D. (2014). Agriculturas familiares y perspectivas hacia el futuro (Agricultures familiales et mondes à venir). J. Sourisseau (ed.). Reseña. Eutopiá, 6, 117-121. https:// doi.org/10.17141/eutopia.6.2014.1602

Martínez, L. y North, L. (2007). El mundo rural en los Andes. Presentación del dossier. Iconos. Revista de Ciencias Sociales, 29, 13-14. https://doi.org/10.17141/iconos.29.2007.234

Martino, D. (2007). Deforestación en la Amazonía: principales factores de presión y perspectivas. Revista del Sur, 169(1), 3-20. Recuperado de http://www.rinconcete. com/files/Deforestacion_amazonia.pdf

Mera, W. y Montaño, V. (1984). Colonización de la región amazónica en el desarrollo capitalista. Quito: Editorial Universitaria UCE.

Ministerio de Agricultura y Ganadería - MAG (1977). La colonización de la Región Amazónica Ecuatoriana. Obra Nacional. Quito: Autor.

Ministerio del Ambiente del Ecuador - MAE (2015). Áreas protegidas del país. [GIS database]. Quito. Recuperado de http://sni.gob.ec/coberturas

Montero, H. y Crespo, C. (1989). Poblaciones Humanas y Desarrollo de la Amazonía Ecuatoriana. En L. Aragón y M. Oliveira (eds.), Populaçôes humanas e desenvolvimento amazônico (pp. 125-192). Belém: Falangola Editora (Série Cooperação Amazônica, 3).

Morrison, K. (2013). Marx, Durkheim, Weber: Formations of Modern Social Thought. 2 da. edición. Londres: Sage.

Pohle, P. (2008). Indigenous and Local Concepts of Land Use and Biodiversity Management in the Andes of Southern Ecuador. En J. Löffler y J. Stadelbauer (eds.), Diversity in mountain systems (pp. 89-105). Colloquium Geographicum 31. Bonn: Departamento de Geografía Universidad de Bonn, Asgard - Verlag Sankt Augustin.

Pohle, P. y Gerique, A. (2008). Sustainable and Non-Sustainable Use of Natural Resources by Indigenous and Local Communities. En E. Beck et al. (eds.), Gradients in a Tropical Mountain Ecosystem of Ecuador (pp. 331-345). Ecological Studies 198. Berlin: Springer Verlag. https://doi.org/10.1007/978-3-540-73526-7_32

Radcliffe, S. (2014). El género y la etnicidad como barreras para el desarrollo: Mujeres indígenas, acceso a recursos en Ecuador en perspectiva latinoamericana. Eutopía, 5, 11-34. https://doi.org/10.17141/eutopia.5.2014.1486

Rivero, S. y Cooney, P. (2010). The Amazon as a Frontier of Capital Accumulation: Looking Beyond the Trees. Capitalist Nature Socialism, 21(4), 50-71. https://doi.org/10 $.1080 / 10455752.2010 .523137$ 
Rudel, T. y Horowitz, B. (1996). La deforestación tropical. Quito: Abya-Yala, Comisión Fullbright y Fundación Jatun Sacha.

Rudel, T., Bates, D. y Machinguiashi, R. (2002). Ecologically Noble Amerindians? Cattle Ranching and Cash Cropping among Shuar and Colonists in Ecuador. Latin American Research Review, 37(1), 144-159. Recuperado de http://www.jstor.org/stable/2692107

Sandner Le Gall, V. (2013). Vidas translocales. Espacios de género y migración ruralurbana indígena en Panamá. En J. Ströbele-Gregor y D. Wollrad (eds.), Espacios de género (pp. 302-317). 1. Edición. Buenos Aires: Nueva Sociedad; Fundación Friedrich Ebert; Adlaf. Recuperado de http://nuso.org/media/documents/Espacios_de_g\%C3\%A9nero_ADLAF_Congreso_anual__2012.pdf

Shukla, J., Nobre, C. y Sellers, P. (1990). Amazon deforestation and climate change. Science, 247(4948), 1322-1325. https://doi.org/10.1126/science.247.4948.1322

Tsing, A. (2005). Friction. An Ethnography of Global Connection. Princeton, Oxford: Princeton University Press. Recuperado de https://books.google.de/books?id=pCwEA1A_XPcC \&printsec $=$ frontcover\&source $=g b s \_g e \_s u m m a r y \_r \& c a d=0 \# v=$ onepage \&q \&f $=$ false

Van der Ploeg, J. (2015). El campesinado y el arte de la agricultura: un manifiesto chayanoviano. Zacatecas: Universidad Autónoma de Zacatecas.

Vásconez, A. (2012). Capítulo II. Caso de Ecuador. En F. Baquero y E. Klein (coords.), Empleo y condiciones de trabajo de mujeres temporeras agricolas (pp. 81-127). Roma: Cepal, FAO, OIT. Recuperado de http://www.fao.org/3/a-as122s.pdf

Villacís, B. y Carrillo, D. (2012). País atrevido: la nueva cara sociodemográfica del Ecuador. Revista Analitika. Quito: INEC.

Vizcarra-Bordi, I., Lutz, B. y Ramírez-Hernández, E. (2013). El mismo fogón: migración y trabajo reproductivo femenino en comunidades mazahuas. Convergencia Revista de Ciencias Sociales, 61, 193-218. Recuperado de https://www.researchgate.net/ publication/262746659_El_mismo_fogon_migracion_y_trabajo_reproductivo_femenino_en_comunidades_mazahuas

Walker, R., Perz, S., Caldas, M. y Silva, L. (2002). Land Use and Land Cover Change in Forest Frontiers: The Role of Household Life Cycles. International Regional Science Review, 25(2), 169-199. https://doi.org/10.1177/016001760202500202

Wasserstrom, R. y Bustamante, T. (2015). Ethnicity, Labor and Indigenous Populations in the Ecuadorian Amazon, 1822-2010. Advances in Anthropology, 5, 1-18. https:// doi.org/10.4236/aa.2015.51001

Yumbla, M. (2014). Fuerza de trabajo femenina en la agricultura de exportación de brócoli en la provincia de Cotopaxi (Tesis de maestría). Departamento de Desarrollo, Ambiente y Territorio, Flacso. Quito. Recuperado de http://repositorio.flacsoandes.edu.ec:8080/ bitstream/10469/7383/2/TFLACSO-2014MRYM.pdf 